ISSN 1794-9831

E-ISSN 2322-7028

Vol. 12 No. 2

Jul - Dic 2015

Cúcuta, Colombia

Recibido:

4 de Diciembre

de 2015

Aceptado por pares:

26 de Diciembre de 2015

Aprobado:

30 de Diciembre de 2015

*Psicóloga Magister en Igualdad y

Género. Profesora

Asociada.

Universidad E1

Bosque. Bogotá,

Colombia. Correo

electrónico:

lafauriemaria@

unbosque.edu.co,

mariamlafaurie $a$

yahoo.com

${ }^{* *}$ Estudiantes de X Semestre de Enfermería.

Universidad El

Bosque. Bogotá,

Colombia. Correo

electrónico:

lcuadros@

unbosque.edu.

co, pagarciab@

unbosque.edu.

co,ihernandez@

unbosque.edu.

co,jpulidoe@

uelbosque.edu.

co, lreynales@

uelbosque.edu.

co, aprodriguez@

uelbosque.edu.

co, psanchezc@

uelbosque.edu.

co,1siastoya@

uelbosque.edu.

co,mtorresri@

uelbosque.edu.co

*** Descriptores

en Ciencias de la

Salud (DeCS), en

la página http://

decs.bvs.br/E/

homepagee.htm

de la Biblioteca

virtual en salud del

proyecto BIREME,

de la Organización

Mundial de la

Salud y de la

Organización

Panamericana de

Salud.

\title{
Violencia de la pareja íntima durante el embarazo y sus repercusiones en la salud mental
}

\author{
María Mercedes Lafaurie-Villamil* \\ Lorena Liseth Cuadros-Salazar* \\ Paola Andrea García-Bocanegra** \\ Ingrid Johana Hernández-Ayala** \\ Johanna Smit Pulido-Espinosa** \\ Lina Fernanda Reynales-Triana** \\ Anyi Paola Rodríguez-Díaz ${ }^{* *}$ \\ Paola Andrea Sánchez-Cortes** \\ Leidy Alejandra Siatoya-Pinto** \\ Mayra Alejandra Torres-Rivera**
}

\section{RESUMEN}

Este artículo presenta un estado del arte sobre violencia de la pareja íntima durante el embarazo y sus implicaciones en la salud mental de la madre, partiendo de 13 artículos publicados entre 2000 y 2014, obtenidos mediante búsqueda en bases de datos (Pubmed, Medline, Nursing Consult, sCielo, Dialnet, Redalyc, Lilacs, Index y Bireme), utilizando como palabras clave: embarazo, violencia de la pareja íntima, salud mental e implicaciones. Los hallazgos de este estudio, muestran que la violencia de la pareja íntima durante el embarazo incrementa la depresión y depresión posparto, así como el malestar emocional en la gestante.

PALABRAS CLAVE: embarazo, género, salud mental, violencia doméstica***

\section{Para citar este artículo / To reference this article / Para citar este artigo}

Lafaurie-Villamil MM, Cuadros-Salazar LL, García-Bocanegra PA, Hernández-Ayala IJ, Pulido-Espinosa JS, Reynales-Triana LF, et al. Violencia de la pareja íntima durante el embarazo y sus repercusiones en la salud mental. Rev. cienc. cuidad. 2015; 12(2): 100116. 


\section{Intimate partner violence during pregnancy and its impact on the mental health}

\section{ABSTRACT}

This article presents a state of the art on intimate partner violence during pregnancy and its implications for the mental health of the mother, based on 13 articles published between 2000 and 2014, they were gotten by looking for databases (PubMed, Medline, Nursing Consult, SciELO, Dialnet, Redalyc, Lilacs, Index and Bireme), using as keywords: pregnancy, intimate partner violence, mental health and implications. The findings of this study show that intimate partner violence during pregnancy increases depression and postpartum depression, and emotional distress in pregnant women.

KEYWORDS: pregnancy, gender, mental health, domestic violence. 



\section{INTRODUCCIÓN}

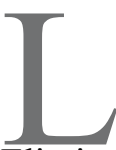

a violencia contra las mujeres constituye una grave violación de los derechos humanos (1). El primer artículo de la Declaración sobre la Eliminación de la Violencia contra la Mujer de las Naciones Unidas, define la violencia de género como "todo acto de violencia basado en la pertenencia al sexo femenino que tenga o pueda tener como resultado un daño o sufrimiento físico, sexual o psicológico para la mujer, incluso las amenazas de tales actos, la coacción o la privación arbitraria de la libertad, tanto si se producen en la vida pública como privada" (2).

Las violaciones a los derechos humanos de las mujeres se relacionan directa o indirectamente con el sistema de género y los valores culturales dominantes (3). La violencia de género, como mecanismo político, busca mantener a las mujeres en desventaja y desigualdad en su relación con el mundo y con los hombres; esto permite excluirlas del acceso a bienes, recursos y oportunidades, además de desvalorizar, denigrar y amedrentar a las mujeres, con lo cual se reproduce el dominio patriarcal (4).

La estrecha relación entre los derechos de las mujeres y su salud sexual y reproductiva, así como la necesidad de afrontar la violencia ejercida contra ellas, son aspectos centrales de la política social de las Naciones Unidas que han sido destacados tanto en la Conferencia de Población y Desarrollo de El Cairo (5), realizada en 1994, como en la IV Conferencia de la Mujer (6) llevada a cabo en Beijing, en 1995.

De acuerdo con las Naciones Unidas (7), la violencia contra las mujeres no se confina a una cultura, región o país específico, ni a grupos particulares de mujeres en la sociedad. Las raíces de la violencia contra la mujer yacen en la discriminación que persiste contra las mujeres.

La violencia de la pareja intima (VPI) y la violencia sexual contra las mujeres representan problemáticas de salud pública de amplia relevancia (1). El estudio de la violencia de la pareja íntima durante el embarazo y sus implicaciones sobre la salud de la gestante representa un campo de indagación de importancia crucial para las disciplinas de la salud, en especial para enfermería (8), y, por lo tanto, fortalecer la comprensión de esta realidad por parte de los profesionales de enfermería redunda en el mejoramiento de la detección, prevención y atención a la gestante víctima de violencia (9).

La violencia de la pareja intima (VPI) durante el embarazo se entiende, según Ramírez (10), como una "situación de coacción y control que incluye la agresión física, sexual o emocional o combinación de ellas y amenazas contra una mujer, por su pareja o expareja, antes, durante y hasta un año después del nacimiento de un hijo". Acerca de las formas que adquiere esta violencia, según su patrón de expresión, Núñez et al. (11) afirman que la VPI se identifica por un patrón de conducta restringida hacia las mujeres que abarca el abuso físico, caracterizado por golpes, quemaduras, mordeduras, heridas con arma blanca o de fuego, mientras que el abuso psicológico y emocional se caracteriza por intimidación, humillaciones verbales, manipulación, omisión, abandono y negligencia, y el abuso sexual consiste en forzar física o psicológicamente a la mujer a tener una relación sexual sin su consentimiento.

La violencia de la pareja íntima en el embarazo es responsable de abortos, óbitos, partos prematuros y otros serios problemas de salud materno-perinatal como el bajo peso al nacer (12). Dentro de las implicaciones de la violencia ejercida por la pareja intima durante el embarazo en esta esfera de la salud materna, los autores reportan, entre otras, depresión y ansiedad (13), depresión postparto (10), estrés postraumático(14) y angustia (15). Cualquier tipo de violencia de la pareja intima contra la mujer gestante genera efectos negativos en su salud mental (13).

Bonifaz y Spano (16) afirman que la violencia psicológica, si bien no deja marcas en el cuerpo, causa un impacto profundo sobre las mujeres, llevando a su destrucción emocional y afectando sus vidas. Según los hallazgos de diversos estudios, la violencia psicológica es la más extendida durante el embarazo (17-21).

De acuerdo con Taillieu et al. (22), el embarazo puede ser un factor protector contra la VPI en un buen número de contextos, lo que no excluye que en ciertos ámbitos representa un serio peligro para las mujeres. Mendoza et al. (23), hallaron en un estudio realizado en México, en que se compararon mujeres embarazadas y no embarazadas victimas de VPI, que es más frecuente la violencia física entre las

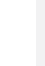


ISSN 1794-9831

E-ISSN 2322-7028

Vol. 12 No. 2

Jul - Dic 2015

Cúcuta, Colombia primeras mientras en las gestantes es más frecuente hallar violencias de tipo psicológico y económico, las cuales se inician o incrementan con la aparición del embarazo.

Las mujeres maltratadas por sus parejas padecen, en mayor medida, problemáticas de salud mental en comparación con las no maltratadas. En el Estudio multipaís de la OMS (24) se registraron más reportes de sufrimiento emocional, ideas suicidas e intentos de suicidio entre las mujeres que alguna vez habían sido víctimas de maltrato físico o sexual que entre las no maltratadas. Además, se han vinculado con la violencia infligida por la pareja los siguientes problemas: abuso de alcohol y de drogas, trastornos de los hábitos alimentarios y del sueño, inactividad física, baja autoestima, trastorno por estrés postraumático, tabaquismo, autoagresión y comportamientos sexuales de riesgo.

Haber tenido una experiencia de violencia doméstica se relaciona estrechamente con depresión perinatal, ansiedad y desorden por estrés postraumático, de acuerdo con una revisión sistemática y metaanálisis conducidos por Howard et al. (25), con base en 67 reportes de investigación.

En un estudio sobre VPI durante el embarazo realizado en Irán por Farrokh et al. (26), en el que participaron 313 puérperas, el 55,9\% informó haber recibido malos tratos por parte de su pareja. Un $43 \%$ de las gestantes experimentaron violencia psicológica en tanto que la violencia física se presentó en un 10.2 $\%$ y la sexual en $17.2 \%$. La baja educación de las parejas y su problemática laboral se destacan entre los factores asociados. Farrokh et al. (26) consideran que en Irán se presentan las tasas de violencia a la gestante más altas del mundo, superando incluso las presentadas en el Mediterráneo Oriental.

En Canadá, Urquia et al. (27) establecieron en un estudio basado en reportes clínicos que, en comparación con las mujeres que no fueron víctimas de abuso en el embarazo, quienes sí lo fueron resultaron ser más propensas a la depresión posparto. Estas mujeres eran en mayor medida jóvenes, solteras, con historias previas de depresión, solían ser amas de casa provenientes de grupos de bajos ingresos y no eran en su mayoría inmigrantes.
Izaguirre et al. (28), en un estudio llevado a cabo en España, establecieron que las víctimas de maltrato y sus hijos no están protegidos contra la violencia durante el embarazo, destacando el impacto de la violencia en las habilidades maternales de las mujeres. El período postnatal parece ser crítico para la seguridad de las víctimas; a menudo, la violencia aumenta después del parto.

Una investigación realizada por Sánchez at al. (29), con 123 gestantes, asistentes a consulta en un centro especializado de México, halló que al menos cuatro de cada 10 mujeres experimentaban violencia conyugal a pesar de encontrarse embarazadas, siendo la mitad de ellas víctimas de más de un tipo de abuso; aquellas que no tenían una pareja estable, estuvieron expuestas en mayor proporción a esa clase de violencia. Se presentó una asociación significativa entre padecer abuso y una sintomatología depresiva de mayor severidad durante el embarazo, así como autoestima baja.

En un estudio adelantado en México por Castro y Ruiz (30), se encontró disminución de la prevalencia de la violencia física que antes del embarazo fue de 17.5 $\%$ y durante el embarazo de $15 \%$. También se halló reducción de la violencia sexual, que pasó de $13.7 \%$ antes del embarazo a $11,8 \%$ durante el embarazo; sin embargo, paradójicamente, la violencia emocional se incrementó: se presentó antes del embarazo en un $23.5 \%$ y durante el embarazo en un $28 \%$, con lo que el embarazo resulta ser un factor de riesgo para desencadenar este tipo de violencia en el contexto estudiado. En el Perú, Tavara-Orozco et al. (31) concluyen igualmente que el embarazo no es un factor protector frente a la VIP.

Mahon et al. (32), en un estudio sobre gestantes provenientes de familias especialmente vulnerables, hallaron una tasa de abuso físico de $1,7 \% \mathrm{y}$ de abuso emocional de $7.5 \%$, aumentando este último, de manera vertiginosa, el trastorno de estrés postraumático. La depresión puede estar presente durante el embarazo pero, al ser maltratadas psicológicamente las mujeres, esta aumenta de forma significativa, según señalan los autores.

Partiendo de un estudio con 268 mujeres en situación de protección, realizado en Suráfrica, Peltzer et al. (33) reportan como consecuencias de la violencia ejercida por la pareja íntima, sobre la salud mental 
de ellas, el trastorno de estrés postraumático y la depresión.

De acuerdo con el análisis de Baird et al. (34), basado en datos de Australia, con frecuencia la violencia psicológica es mucho más fuerte que el abuso físico al que son expuestas las mujeres por sus parejas, siendo esta forma de violencia precursora de la violencia física, por lo que puede llegar a tener gran impacto sobre la salud de la mujer, comprometiendo sobre todo su salud mental. Se estableció que las mujeres que están deprimidas durante el embarazo son más propensas a experimentar algún tipo de depresión en el posparto.

Se identifican dos tipos de reacciones depresivas después del parto: a) el blues materno y b) la depresión postparto. De acuerdo con Baird et al. (34), las mujeres más jóvenes (entre 18 y 24 años) son quienes están más propensas a recibir violencia por parte de sus parejas durante la gestación. De otra parte, en un estudio realizado en Brasil, en el año 2014, por Ludermir et al (35), se encontró que la prevalencia de los trastornos mentales entre las mujeres que refirieron violencia durante el embarazo alcanzaba el 71,0\%.

Las grandes diferencias, en cuanto a la prevalencia y los patrones de violencia, encontradas de un país a otro -y sobre todo de un entorno a otro dentro de los diferentes países-, indican que no hay nada que sea natural o inevitable sobre este problema. Las actitudes pueden y requieren modificarse, las condiciones de las mujeres pueden y deben mejorarse, tanto hombres como mujeres pueden y deben convencerse de que la violencia infligida por la pareja no puede aceptarse en una relación humana (24).

Dado que los estudios sobre violencia a la mujer constituyen un área de investigación muy reciente, la mayoría de los trabajos publicados hacen referencia a la violencia en general o sólo a la violencia física, ya que es la de mayor visibilidad y más fácil de cuantificar, y hay muy poca información sobre la frecuencia y características de otras formas de maltrato (36).

Partiendo de la importancia que tiene aportar información relevante para fortalecer la construcción de lineamientos para la detección, prevención y atención a la gestante víctima de violencia, desde la disciplina de enfermería, se construyó un estado del arte sobre violencia de la pareja intima en el embarazo y sus repercusiones en la salud mental de la gestante, en el cual se analizaron productos del conocimiento circulado sobre este tópico (37) y se describieron tendencias y hallazgos de la investigación (38).

Este proceso de indagación fue construido partiendo de una búsqueda en torno a la literatura científica en español e inglés, producida en diferentes regiones del mundo entre 2000 y 2014, teniendo en cuenta bases de datos de salud como Pubmed, Medline, Nursing Consult, sCielo, Dialnet, Redalyc, Lilacs, Index y Bireme. Como criterios de inclusión se establecieron los siguientes: artículos que reporten resultados de estudios publicados entre 2000 y 2014, que ofrezcan información científica sobre las repercusiones de la VPI en la salud mental de la gestante. Los siguientes descriptores facilitaron la búsqueda: embarazo, violencia de la pareja íntima, salud mental e implicaciones.

Se seleccionaron 13 artículos, provenientes de ocho países, que cumplieron con los criterios de inclusión, 12 de tipo cuantitativo y uno de perspectiva cualitativa, primando entre ellos los estudios de corte transversal de carácter prospectivo.

Se implementó un análisis documental, llevando a cabo una revisión a fondo de cada fuente seleccionada y utilizando la técnica de fichaje propuesta por Montero y Hachman para, posterior a la recolección de información relevante, proceder al desarrollo de un análisis crítico (39).

Según la Resolución 008430 de 1993, del Ministerio de Salud (40), se considera sin riesgo la investigación de tipo documental. Se observaron los principios expuestos por la Ley 911 de 2004 de la Republica de Colombia, Capítulo IV del Título III, acerca de la calidad ética de la investigación realizada en enfermería, en la que es un requisito, entre otros, el respeto a la propiedad intelectual (41). Se tuvieron en consideración los derechos de autor en todos los productos analizados, respetando las normas de referenciación.

Se presenta en los siguientes apartes, con apoyo de tablas descriptivas, la información más destacada que fue obtenida en el proceso de exploración realizado. 
ISSN 1794-9831

E-ISSN 2322-7028

Vol. 12 No. 2

Jul - Dic 2015

Cúcuta, Colombia

\section{Sobre el origen de los estudios analizados}

Como se puede ver en la tabla 1, los estudios consultados provienen de los siguientes países: China (42-43), India (44), Suráfrica (45), Tanzania (46),
Perú (47-48), Brasil (35, 49-51), Costa Rica (11) y Colombia (10). Brasil cuenta con amplia producción en este campo, teniéndose en cuenta 5 artículos realizados en ese país.

Tabla 1. Artículos analizados por países.

\begin{tabular}{|c|c|c|c|}
\hline Región & País & $\begin{array}{l}\text { Artículos } \\
\text { Analizados }\end{array}$ & Autores \\
\hline \multirow{3}{*}{ Asia y el Pacifico } & & \multirow[b]{2}{*}{2} & Tiwari et al. (2008) (42) \\
\hline & China & & Zhang et al. (2012) (43) \\
\hline & India & 1 & Varma et al. (2007) (44) \\
\hline \multirow[b]{2}{*}{ África } & Suráfrica & 1 & Groves et al. (2012) (45) \\
\hline & $\begin{array}{l}\text { República Unida de } \\
\text { Tanzania }\end{array}$ & 1 & Mahengue et al. (2013) (46) \\
\hline \multirow{8}{*}{$\begin{array}{l}\text { América Latina y el } \\
\text { Caribe }\end{array}$} & & & Lam et al. (2008) (47) \\
\hline & Perú & 2 & Gómez et al. (2009) (48) \\
\hline & \multirow{4}{*}{ Brasil } & \multirow{4}{*}{5} & Ludemir et al. (35) \\
\hline & & & Cowley(2010) (49) \\
\hline & & & Nunes et al. (2010) (50) \\
\hline & & & Santos et al. (2014) (51) \\
\hline & Costa Rica & 1 & Núñez et al. (2003) (11) \\
\hline & Colombia & 1 & Ramírez (2012) (10) \\
\hline \multicolumn{2}{|l|}{ Total } & 13 & \\
\hline
\end{tabular}

Fuente: Elaboración propia.

Principales repercusiones de la violencia de pareja sobre la salud mental de la gestante

En la tabla 2 se describen las repercusiones más destacadas de la VPI sobre la presentación de problemáticas de salud mental, teniendo en cuenta el tipo de violencia a la que las gestantes han estado expuestas. Entre las alteraciones y trastornos de conducta más frecuentemente reportados por los autores consultados, en relación con la violencia de la pareja intima durante el embarazo, se encuentran la depresión, la depresión postparto, el trastorno de estrés postraumático y el síndrome de estrés postraumático. Resultados similares han sido hallados por Howard (25) en su trabajo de revisión sistemática sobre violencia domestica y desórdenes perinatales de salud mental.
Lam et al. (47) hallaron que las gestantes maltradas tuvieron 4.37 veces más riesgo de presentar depresión gestacional que aquellas no maltratadas. Es importante destacar que la depresión conlleva, entre otras consecuencias, disminución del apetito y alteración del sueño, acompañados de autovaloración negativa, ideas de culpa e inutilidad, falta de interés y de concentración, disminución de la energía y cansancio (52).

En el embarazo, la depresión puede verse reflejada en cambios comportamentales como el abandono de los controles prenatales, la falta de autocuidado que debe ser propia de la gravidez, la falta de adherencia a las recomendaciones médicas y el abuso de tabaco, alcohol y sustancias psicoactivas, lo que puede afectar el desenlace obstétrico (53) 
La depresión posparto afecta el estado de ánimo de la mujer y puede tener inicio en cualquier momento durante el primer año después del parto. Se manifiesta como una pérdida de control de las emociones, pensamientos y acciones $\mathrm{y}$, además, conlleva síntomas que pueden incluir ansiedad, incapacidad para concentrarse, sentimientos de soledad e inseguridad, dificultad en el dormir o comer, culpa, vergüenza, pensamiento obsesivo e ideas relacionadas con lesionarse a sí misma o al bebé (9).

En cuanto al trastorno de estrés postraumático (TEPT), se trata de "una de las formas del estrés humano más incapacitantes" (54), con implicaciones a nivel familiar, laboral y social; datos de origen psicológico y biológico sugieren que deviene de un fracaso en el control del estrés durante el momento del trauma (54).

El TEPT implica que la persona haya estado expuesta a la muerte, lesión grave o violencia sexual, sea real o amenaza. El acontecimiento es reexperimentado de modo persistente por la mujer, lo que la lleva a evitar estímulos asociados; se alteran negativamente la cognición y el estado de ánimo, se presentan trastornos importantes de la alerta y reactividad y malestar clínico significativo que afecta la vida laboral y social, no atribuibles a alguna sustancia, durante un mes o más, de acuerdo con el DSMV (Diagnostic and statistical manual of mental disorders) (55).

Cuando el trastorno por estrés postraumático se ve asociado a violencia doméstica o intrafamiliar, las mujeres pueden vivir la maternidad como una amenaza para su integridad física y los procedimientos ginecológicos, con frecuencia, reactivan la sintomatología. EL TEPT implica riesgos en la gestación como preeclampsia y requerimiento de cesárea, además de afectar la calidad de la relación madre-hijo (54).

Es importante mencionar que, por las características de los estudios analizados, no es posible establecer una relación causal entre la VPI en el embarazo y la existencia de trastornos y alteraciones de salud mental de la gestante; sin embargo, los avances analizados afirman la idea de que estas problemáticas se ven incrementadas con la presencia de esta forma de violencia.
En lo relativo a los tipos de violencia de la pareja íntima y su relación con problemáticas de salud mental en la gestante, aparecen datos como los obtenidos por Mahengue et al. (46), quienes reportan que la depresión se ve mayormente asociada a las violencia física y sexual que a la violencia psicológica por parte de la pareja íntima, a partir de un estudio con gestantes de Tanzania. De otra parte, los estudios de Nunes et al. (50) y Santos et al. (51), realizados en el Brasil, reportan que esta problemática se ve estrechamente relacionada con la violencia psicológica.

Cowley (49), en su estudio realizado en Brasil, evaluó la incidencia de la violencia psicológica en la presentación de trastornos y expresiones de malestar emocional en las gestantes con VPI. Encontró que cuando se presenta violencia de tipo físico y sexual, además de la violencia psicológica, es mayor la posibilidad de que se presente depresión posparto; no osbtante, de acuerdo con esta autora, la violencia psicológica puede incrementar progresivamente la presentación de síntomas depresivos.

Zhang et al. (43) señalan, a partir de un estudio realizado en China, que la depresión posparto se ve asociada sobre todo a la violencia psicológica durante el embarazo. Similares resultados se observan en el estudio de Tiwari et al. (42), realizado en la misma región.

La violencia de la pareja íntima, de tipo físico, sexual o psicológico, impacta negativamente en la salud mental de la gestante, pero, como lo menciona Cowley (41), la violencia psicológica por sí misma -sin que se presenten necesariamente otros tipos de expresiones violentas-, puede desencadenar expresiones de malestar emocional.

Ahora bien, los hallazgos obtenidos, más que conducir a hipótesis sobre la posible relación entre tipo de violencia de la pareja íntima y determinadas problemáticas de salud mental, deben ser leídos a la luz de las particularidades culturales propias de cada contexto. Igualmente, es necesario tener en cuenta que, durante el embarazo, se pueden inhibir expresiones de violencia de la pareja íntima de tipo físico y sexual, permaneciendo sobre todo la violencia psicológica.
ISSN 1794-9831

E-ISSN 2322-7028

Vol. 12 No. 2

Jul - Dic 2015

Cúcuta, Colombia

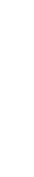


Violencia de la pareja íntima durante el embarazo y sus repercusiones en la salud mental • María Mercedes Lafaurie-Villamil, Lorena Liseth Cuadros-Salazar, Paola Andrea García-Bocanegra, Ingrid Johana Hernández-Ayala, Johanna Smit Pulido-Espinosa, Lina Fernanda Reynales-Triana, et al.

ISSN 1794-9831

E-ISSN 2322-7028

Vol. 12 No. 2

Jul - Dic 2015

Cúcuta, Colombia
En un estudio analítico (12) sobre VPI en el embarazo, publicado en 2015, se estableció que la violencia de la pareja íntima de tipo psicológico suele prevalecer, durante la gestación, sobre las demás formas de violencia. Las formas que adquiere este tipo de violencia a las gestantes suelen estar relacionadas con los ámbitos sociales y culturales en que se presentan.
Resulta de importancia crucial el reconocimiento de la violencia psicológica como un aspecto que reviste gran relevancia a la hora de minimizar la incidencia de problemáticas como la depresión durante la gestación.

Tabla 2. Repercusiones de la VPI en la presentación de problemáticas de salud mental en la gestante.

\begin{tabular}{|c|c|c|c|c|}
\hline Repercusiones & Tipo de violencia & Autor(es)/Año & País & Región \\
\hline \multirow{6}{*}{$\begin{array}{l}\text { En la presentación de } \\
\text { depresión }\end{array}$} & $\begin{array}{ll}- & \text { Física } \\
- & \text { Psicológica } \\
- & \text { Sexual } \\
\end{array}$ & $\begin{array}{l}\text { Núñez et al. } \\
\text { (2003) (11) }\end{array}$ & Costa Rica & $\begin{array}{l}\text { América } \\
\text { Central }\end{array}$ \\
\hline & - $\quad$ Psicológica & $\begin{array}{l}\text { Nunes et al. } \\
(2010)(50)\end{array}$ & Brasil & $\begin{array}{l}\text { América del } \\
\text { sur }\end{array}$ \\
\hline & $\begin{array}{ll}- & \text { Física } \\
- & \text { Sexual } \\
\end{array}$ & Mahenge et al. (2013) (46) & Tanzania & África \\
\hline & $\begin{array}{ll}- & \text { Psicológica }\end{array}$ & $\begin{array}{l}\text { Santos et al. } \\
(2014)(51)\end{array}$ & Brasil & $\begin{array}{l}\text { América del } \\
\text { sur }\end{array}$ \\
\hline & $\begin{array}{ll}- & \text { Física } \\
- & \text { Sexual } \\
- & \text { Psicológica } \\
\end{array}$ & Gómez et al. (2009) (48) & Perú & $\begin{array}{l}\text { América del } \\
\text { sur }\end{array}$ \\
\hline & $\begin{array}{ll}- & \text { Física } \\
- & \text { Psicológica } \\
- & \text { Sexual } \\
\end{array}$ & Lam et al. (2008) (47) & Perú & $\begin{array}{l}\text { América del } \\
\text { sur }\end{array}$ \\
\hline \multirow{4}{*}{$\begin{array}{l}\text { En la presentación de } \\
\text { depresión posparto }\end{array}$} & Psicológica & Zhang et al. (2012) (43) & Hong Kong & Asia \\
\hline & - $\quad$ Psicológica & $\begin{array}{l}\text { Tiwari et al. } \\
(2008)(42)\end{array}$ & China & Asia \\
\hline & $\begin{array}{ll}- & \text { Física } \\
- & \text { Sexual } \\
- & \text { Psicológica }\end{array}$ & $\begin{array}{l}\text { Cowley } \\
(2010)(49)\end{array}$ & Brasil & $\begin{array}{l}\text { América del } \\
\text { sur }\end{array}$ \\
\hline & $\begin{array}{ll}- & \text { Física } \\
- & \text { Sexual } \\
- & \text { Psicológica } \\
\end{array}$ & Ludemir et al. (2010) (35) & Brasil & $\begin{array}{l}\text { América del } \\
\text { sur }\end{array}$ \\
\hline \multirow{3}{*}{$\begin{array}{l}\text { En presentación de } \\
\text { TEPT (trastorno de } \\
\text { estrés postraumático) }\end{array}$} & $\begin{array}{ll}- & \text { Física } \\
- & \text { Sexual } \\
\end{array}$ & Mahenge et al. (2013) (46) & Tanzania & África \\
\hline & $\begin{array}{ll}- & \text { Psicológica }\end{array}$ & $\begin{array}{l}\text { Varma et al. } \\
(2006)(44) \\
\end{array}$ & India & Asia \\
\hline & $\begin{array}{ll}- & \text { Psicológica }\end{array}$ & Santos et al. (2014) (51) & Brasil & $\begin{array}{l}\text { América del } \\
\text { sur }\end{array}$ \\
\hline
\end{tabular}

Fuente: Elaboración propia.

\section{Expresiones de malestar emocional relacionadas con la violencia de pareja íntima a la gestante}

Si bien es cierto que la presentación de expresiones de malestar emocional en el embarazo resulta ser un fenómeno multifactorial, se encuentra que la violencia de la pareja íntima a la gestante incrementa sustancialmente las posibilidades de que estas manifestaciones se exacerben. En la tabla 3 se dan a conocer las principales repercusiones de la VPI en el embarazo sobre la presentación de expresiones de malestar emocional en la gestante. 
Tabla 3. Repercusiones de la VPI en el embarazo sobre la presentación de expresiones de malestar emocional.

\begin{tabular}{|c|c|c|c|c|}
\hline Repercusiones & Tipo de violencia & Autor(es)/Año & País & Región \\
\hline \multirow[b]{5}{*}{ En la presentación de ansiedad } & $\begin{array}{ll}- & \text { Física } \\
- & \text { Psicológica } \\
- & \text { Sexual }\end{array}$ & Núñez et al. (2003) (11) & Costa Rica & América Central \\
\hline & - $\quad$ Psicológica & Ludermir et al. (2010) (35) & Brasil & América del sur \\
\hline & - $\quad$ Psicológica & $\begin{array}{l}\text { Ramírez } \\
(2012)(10)\end{array}$ & Colombia & América del sur \\
\hline & $\begin{array}{ll}\text { - } & \text { Física } \\
\text { - } & \text { Sexual }\end{array}$ & $\begin{array}{l}\text { Mahenge et al. } \\
(2013)(46)\end{array}$ & Tanzania & África \\
\hline & $\begin{array}{ll} & \text { Psicológica }\end{array}$ & $\begin{array}{l}\text { Santos et al. } \\
(2014)(51)\end{array}$ & Brasil & América del sur \\
\hline \multirow[t]{2}{*}{$\begin{array}{l}\text { En la presentación de ideas } \\
\text { suicidas }\end{array}$} & - $\quad$ Psicológica & $\begin{array}{l}\text { Ramírez } \\
(2012)(10)\end{array}$ & Colombia & América del sur \\
\hline & - $\quad$ Psicológica & Santos et al. (2014) (51) & Brasil & América del sur \\
\hline \multirow[t]{2}{*}{$\begin{array}{l}\text { En la presentación de baja } \\
\text { autoestima }\end{array}$} & $\begin{array}{ll}- & \text { Física } \\
- & \text { Psicológica } \\
- & \text { Sexual }\end{array}$ & $\begin{array}{l}\text { Núñez et al. } \\
\text { (2003) (11) }\end{array}$ & Costa Rica & América Central \\
\hline & - $\quad$ Psicológica & $\begin{array}{l}\text { Ramírez } \\
(2012)(10)\end{array}$ & Colombia & América del sur \\
\hline $\begin{array}{l}\text { En la presentación de síntomas } \\
\text { depresivos }\end{array}$ & Psicológica & $\begin{array}{l}\text { Ramírez } \\
(2012)(10)\end{array}$ & Colombia & América del sur \\
\hline $\begin{array}{l}\text { En la presentación labilidad } \\
\text { emocional }\end{array}$ & Psicológica & $\begin{array}{l}\text { Ramírez } \\
(2012)(10)\end{array}$ & Colombia & América del sur \\
\hline \multirow{2}{*}{ En la presentación de angustia } & $\begin{array}{ll}- & \text { Física } \\
- & \text { Psicológica } \\
- & \text { Sexual }\end{array}$ & $\begin{array}{l}\text { Núñez et al. } \\
\text { (2003) (11) }\end{array}$ & Costa Rica & América Central \\
\hline & Psicológica & Groves et al. (45) & Suráfrica & África \\
\hline $\begin{array}{l}\text { En la presentación de insegu- } \\
\text { ridad }\end{array}$ & - $\quad$ Psicológica & $\begin{array}{l}\text { Ramírez } \\
(2012)(10)\end{array}$ & Colombia & América del sur \\
\hline En la presentación de miedo & $\begin{array}{ll}- & \text { Física } \\
- & \text { Psicológica } \\
- & \text { Sexual }\end{array}$ & $\begin{array}{l}\text { Núñez et al. } \\
\text { (2003) (11) }\end{array}$ & Costa Rica & América Central \\
\hline $\begin{array}{l}\text { En la presentación de senti- } \\
\text { mientos de culpa/o vergüenza }\end{array}$ & - $\quad$ Psicológica & $\begin{array}{l}\text { Ramírez } \\
(2012)(10)\end{array}$ & Colombia & América del sur \\
\hline $\begin{array}{l}\text { En la presentación de estrés } \\
\text { materno }\end{array}$ & $\begin{array}{ll}- & \text { Física } \\
- & \text { Psicológica } \\
- & \text { Sexual }\end{array}$ & Nunes et al. (2010) (50) & Brasil & América del sur \\
\hline $\begin{array}{l}\text { En la presentación de tristeza } \\
\text { postnatal (o baby blues) }\end{array}$ & Psicológica & $\begin{array}{l}\text { Ramírez } \\
(2012)(10)\end{array}$ & Colombia & América del sur \\
\hline
\end{tabular}

Fuente: Elaboración propia.

El estudio llevado a cabo en Colombia por Ramírez (10) reporta que la VPI se relaciona sobre todo con sintomatología depresiva: labilidad emocional, ansiedad e inseguridad están entre las dimensiones más afectadas. Este estudio también descubrió que la violencia de tipo psicológico acrecienta la aparición de tristeza postnatal o baby blues, siendo considerada ésta como algo normal durante el puerperio, por ser de muy corta duración y de leve sintomatología. Tal cual lo propone Baird (34), la depresión posparto y la tristeza posnatal pueden verse incrementadas con el maltrato de la pareja íntima durante la gestación, a pesar de ser trastornos propios de la etapa puerperal de la mujer.

Tres de los estudios realizados en Brasil, en las ciudades de Porto Alegre (50), Recife (35) y Salvador de Bahía (49) y el estudio realizado en Tanzania (46) 
ISSN 1794-9831

E-ISSN 2322-7028

Vol. 12 No. 2

Jul - Dic 2015

Cúcuta, Colombia coinciden en que una presentación muy frecuente en las mujeres víctimas de VPI en el embarazo es la ansiedad. La ansiedad extrema en el embarazo produce un gran sufrimiento a la madre y puede causar problemas en el desarrollo fetal, además de afectar la morfología y la fisiología del feto. De igual forma, puede generar cambios en el comportamiento y estado emocional del bebé e incidir en el bajo peso al nacer (56).

La presentación de baja autoestima en las gestantes con VPI, reportada por Nuñez et al. (11) y Ramírez (10), también ha sido reconocida por Mendoza et al. (23), quienes encontraron que esta situación constituye un factor de riesgo para la presentación de violencias de género en el embarazo, y por Sánchez et al. (29), quienes hallaron relación entre padecer abuso, mostrar una sintomatología depresiva de mayor severidad durante el embarazo y presentar autoestima baja. La autoestima habla de sentimientos como la aceptación y aprecio hacia sí mismo, lo cual se relaciona con el sentimiento de competencia y de valía personal (57).

La afectación de esta dimensión de la identidad incide en la salud de las mujeres y se ve relacionada con la forma de afrontamiento del estrés, con el control de las emociones y con la depresión (58). Y es que, según lo exponen Ludemir et al. (35), la agresión por parte de alguien con quien se está emocionalmente involucrado puede llevar a desarrollar sentimientos de impotencia, baja autoestima y depresión.

El hecho de recibir violencia psicológica parece tener relación estrecha con la presentación de prácticamente todas las expresiones de malestar emocional en las gestantes víctimas de violencia de la pareja íntima mencionadas por los autores cuyos estudios han sido analizados aquí.

\section{Factores asociados a la afectación de la salud mental en la gestante con violencia de la pareja íntima}

Algunos estudios exploraron factores asociados a la presentación de problemáticas de salud mental en mujeres víctimas de violencia de la pareja íntima en el embarazo (especialmente depresión y depresión postparto). En la la tabla 4 se describen estos factores, destacándose, entre otros aspectos, que la vivencia de violencia psicológica y los antecedentes de violencia de género, así como aspectos asociados a las precarias condiciones sociales de la gestante, tienen un peso especial para que se vean incrementadas estas manifestaciones. 
Tabla 4. Factores asociados a la presentación de problemáticas de salud mental en gestantes con VPI.

ISSN 1794-9831

E-ISSN 2322-7028

\begin{tabular}{|c|c|c|c|c|}
\hline Factores asociados & Se asocia a & Autor(es)/Año & País & Región \\
\hline \multirow{4}{*}{ Violencia psicológica } & - $\quad$ Depresión postparto & Tiwari et al. (2008) (42) & Hong Kong & Asia \\
\hline & $\begin{array}{ll}- & \text { Depresión posparto }\end{array}$ & Zhang et al. (2012) (43) & China & Asia \\
\hline & $\begin{array}{ll}\text { - } & \text { Depresión postparto } \\
\text { - } & \text { Depresión }\end{array}$ & $\begin{array}{l}\text { Ludermir et al. } \\
\text { (2010) (35) }\end{array}$ & Brasil & América del sur \\
\hline & - $\quad$ Depresión postparto & $\begin{array}{l}\text { Nunes et al. } \\
(2010)(50)\end{array}$ & Brasil & América del sur \\
\hline $\begin{array}{l}\text { El abuso sexual en el año } \\
\text { anterior }\end{array}$ & Depresión & Zhang et al. (2012) (43) & China & Asia \\
\hline $\begin{array}{l}\text { Una actitud de aceptación } \\
\text { hacia la violencia como algo } \\
\text { inevitable }\end{array}$ & Depresión & Zhang et al. (2012) (43) & China & Asia \\
\hline El embarazo inesperado & - $\quad$ Depresión & Zhang et al. (2012) (43) & China & Asia \\
\hline $\begin{array}{l}\text { Un estilo de afrontamiento } \\
\text { negativo }\end{array}$ & Depresión postparto & Zhang et al. (2012) (43) & China & Asia \\
\hline $\begin{array}{l}\text { La preocupación por la salud } \\
\text { del bebé }\end{array}$ & Depresión & Zhang et al. (2012) (43) & China & Asia \\
\hline $\begin{array}{l}\text { Mujeres que no viven con su } \\
\text { pareja }\end{array}$ & Depresión & $\begin{array}{l}\text { Ludemir et al. } \\
(2010)(35)\end{array}$ & Brasil & América del sur \\
\hline $\begin{array}{l}\text { Mujeres con } 4 \text { años de } \\
\text { educación o menor a esta }\end{array}$ & Depresión & $\begin{array}{l}\text { Ludemir et al. } \\
(2010)(35)\end{array}$ & Brasil & América del sur \\
\hline Mujeres desempleadas & Depresión & $\begin{array}{l}\text { Ludemir et al. } \\
(2010)(35)\end{array}$ & Brasil & América del sur \\
\hline $\begin{array}{l}\text { Mujeres con mala relación } \\
\text { de pareja }\end{array}$ & Depresión & $\begin{array}{l}\text { Ludemir et al. } \\
(2010)(35)\end{array}$ & Brasil & América del sur \\
\hline \multirow[t]{2}{*}{ Poco o nada de apoyo social } & - $\quad$ Depresión & $\begin{array}{l}\text { Ludemir et al. } \\
(2010)(35)\end{array}$ & Brasil & América del sur \\
\hline & Depresión & Núñez et al (2011) & Costa Rica & América Central \\
\hline $\begin{array}{l}\text { Mujeres con alguna } \\
\text { enfermedad mental antes o } \\
\text { durante el embarazo }\end{array}$ & Depresión & $\begin{array}{l}\text { Ludemir et al. } \\
(2010)(35)\end{array}$ & Brasil & América del sur \\
\hline $\begin{array}{l}\text { Dos o más exposiciones de } \\
\text { violencia durante el } \\
\text { embarazo }\end{array}$ & Estrés materno & $\begin{array}{l}\text { Nunes et al. } \\
(2010)(50)\end{array}$ & Brasil & América del sur \\
\hline $\begin{array}{l}\text { Poca atención prenatal y } \\
\text { nutrición deficiente }\end{array}$ & $\begin{array}{l}\text { Depresión durante } \\
\text { el embarazo }\end{array}$ & $\begin{array}{l}\text { Nunes et al. } \\
(2010)(50)\end{array}$ & Brasil & América del sur \\
\hline Fumar durante el embarazo & $\begin{array}{l}\text { Depresión durante } \\
\text { el embarazo }\end{array}$ & $\begin{array}{l}\text { Nunes et al. } \\
(2010)(50)\end{array}$ & Brasil & América del sur \\
\hline $\begin{array}{l}\text { Ingesta de alimentos } \\
\text { deficiente }\end{array}$ & Trastorno depresivo & $\begin{array}{l}\text { Nunes et al. } \\
(2010)(50)\end{array}$ & Brasil & América del sur \\
\hline $\begin{array}{l}\text { Antecedente de violencia } \\
\text { durante embarazo }\end{array}$ & Depresión & Lam et al. (2008) (47) & Perú & América del sur \\
\hline $\begin{array}{l}\text { Bajos niveles de resiliencia y } \\
\text { apoyo social }\end{array}$ & Depresión & Lam et al. (2008) (47) & Perú & América del sur \\
\hline $\begin{array}{l}\text { Antecedente de Violencia de } \\
\text { género }\end{array}$ & Depresión & Lam et al. (2008) (47) & Perú & América Latina \\
\hline \multirow{2}{*}{$\begin{array}{l}\text { El consumo excesivo de } \\
\text { alcohol por parte de la mujer } \\
\text { gestante }\end{array}$} & $\begin{array}{ll}- & \text { Depresión }\end{array}$ & Zhang et al. (2012) (43) & China & Asia \\
\hline & Depresión postparto & $\begin{array}{l}\text { Nunes et al. } \\
(2010)(50)\end{array}$ & Brasil & América del sur \\
\hline \multirow{2}{*}{$\begin{array}{l}\text { El consumo excesivo de } \\
\text { alcohol por parte de la pareja }\end{array}$} & Depresión & Zhang et al. (2012) (43) & China & Asia \\
\hline & - $\quad$ Depresión postparto & $\begin{array}{l}\text { Nunes et al. } \\
(2010)(50)\end{array}$ & Brasil & América del sur \\
\hline
\end{tabular}

Fuente: Elaboración propia. 
ISSN 1794-9831

E-ISSN 2322-7028

Vol. 12 No. 2

Jul - Dic 2015

Cúcuta, Colombia
La experiencia de violencia sexual en el año precedente al embarazo, el embarazo no deseado, la actitud de aceptación frente a la violencia y el consumo excesivo de alcohol por parte de la pareja son aspectos que pueden precipitar la depresión postparto, según Zhang et al. (43). La aceptación de la violencia como algo inherente a la vida de las mujeres -que es inevitable-, impide que ellas tomen posición frente al maltrato que reciben de sus parejas. Las actitudes de aceptación de la violencia representan un factor de riesgo de violencia contra las mujeres destacado por la Organización Mundial de la Salud (59).

Las condiciones difíciles en que se vive el embarazo, tales como la mala alimentación y los hábitos perjudiciales (fumar o beber en exceso), así como la inasistencia a controles prenatales, se asocian igualmente a la presentación de problemáticas de salud mental relacionadas con VPI a la gestante, de acuerdo con el análisis realizado por Nunes (50).

Por otro lado, el hecho de no vivir con la pareja, tener baja escolaridad y estar desempleada, precipita la depresión en gestantes con VPI, de acuerdo con Ludemir et al. (35). La falta de apoyo expresada por las gestantes se ve también relacionada con la depresión. Lam y sus colaboradores (47) encontraron que la depresión gestacional suele ser más frecuente cuando las mujeres víctimas de VPI poseen bajos niveles de resiliencia y de apoyo social; quienes presentaban adecuado apoyo social, mostraban un riesgo 79 \% menor de presentar depresión gestacional; las gestantes resilientes presentaban un $41 \%$ menos de probabilidades. En el estudio realizado por Nuñéz et al. (11) "se detectaron diferencias estadísticamente significativas entre las mujeres que se sentían deprimidas y las que se sentían apoyadas".

\section{Alternativas de intervención}

En los estudios analizados se proponen alternativas de intervención que pueden ser útiles para la detección y la atención a la gestante víctima de violencia. Teniendo en cuenta la relevancia que tiene el poder contar con algunos referentes en este campo, al ser esta una revisión que surge dentro del contexto de enfermería, se describirán algunas de ellas.

Tiwari et al. (42) advierten que el embarazo ofrece una ventana de oportunidad para que los profesionales de la salud puedan ayudar a las mujeres que son víctimas de abusos psicológicos. En cuanto a la detección de la VPI en el embarazo, el estudio realizado en Colombia por Ramírez (10) señala que el uso de instrumentos que permitan la identificación rápida y adecuada de los casos y la utilización de los predictores de depresión postparto de Sheryl Tatano Beck, sería de gran beneficio, pudiendo ser el enfermero quien utilice sus habilidades de comunicación terapéutica y de escucha como intervenciones iniciales en los casos donde se presenten situaciones de violencia.

Nuñez et al. (11) exponen que los especialistas no sólo deben enfocarse en el abordaje a la violencia física o sexual ejercida a las mujeres durante el embarazo, sino que deben explorar la violencia psicológica y evaluar el estado emocional de las gestantes. De acuerdo con estos autores, ello permite dimensionar tanto el estrés postraumático como el desequilibrio psicológico-emocional y el apoyo psicosocial, con lo que será posible "diseñar y desarrollar estrategias de intervención a favor de las mujeres en mayor riesgo" (11).

Varma et al. (44) señalan que generar una comprensión adecuada de las manifestaciones de síntomas presentados por las mujeres gestantes que visitan frecuentemente las instituciones de salud, ofrece oportunidades a los proveedores de atención médica de explorar la historia de maltrato dentro de la familia. También sugieren aclarar, en mayor medida, la relación entre las diferentes formas de VPI (psicológica, física y sexual) y los resultados de salud mental, especialmente en los países en vía de desarrollo. Al respecto, Zhang y sus colaboradores (43) recalcan, igualmente, la importancia de la intervención comunitaria en las poblaciones vulnerables de los países de bajos ingresos.

De otra parte, Mahenge et al. (46), a partir de su estudio en Tanzania y ante las alarmantes implicaciones de la VPI sobre la salud mental de las gestantes, destacan la importancia de formar y educar a los proveedores de atención prenatal para crear conciencia de esta realidad.

Ludemir et al. (35), señalan que, más allá de detectar la violencia en el embarazo dentro del contexto de la atención prenatal, se requiere contar con redes de apoyo efectivo para las mujeres víctimas de maltrato, que ofrezcan alternativas de protección, asesoría de tipo legal y apoyo psicológico. Insisten en la 
importancia de contar con protocolos de intervención para el empoderamiento de las mujeres.

\section{CONCLUSIONES}

A partir de la revisión realizada, se establece la gran importancia de comprender las repercusiones que puede tener la VPI durante el embarazo sobre la salud mental de la gestante y de detectar dichas situaciones a tiempo en el contexto de la salud. En el embarazo, la mujer está expuesta a situaciones de riesgo, siendo vital detectar factores que puedan incidir en que la madre se vea enfrentada a consecuencias negativas para su salud.

Los hallazgos obtenidos mediante este estado del arte evidencian circunstancias como la existencia de violencia psicológica que, a la luz de la revisión bibliográfica, acrecientan la presentación de alteraciones en la salud mental de la gestante víctima de VPI, en las cuales prima la depresión, la depresión postparto y el trastorno de estrés postraumático, así como diversas expresiones de malestar emocional dentro de las cuales se destaca la ansiedad.

Bajos niveles de apoyo social, poca capacidad de las mujeres para enfrentar el abuso y afectación de su calidad de vida, de su capacidad para la toma de decisiones en salud sexual y reproductiva y de su autocuidado, son aspectos que incrementan la presentación de tratornos y alteraciones de la salud mental cuando existe violencia de la pareja íntima durante la gestación.

Es válido destacar la estrecha relación entre la VPI, la presentación de problemáticas de salud mental y la existencia de riesgos del embarazo con problemas relativos a la ingesta de alimentos, la poca asistencia a controles prenatales y los hábitos nocivos para la salud de las gestantes.

El presente artículo permite reflexionar sobre la importancia de explorar en las gestantes en quienes se presentan estas circunstancias, la posible presentación de VPI, con el fin de prevenir en ellas el deterioro de su salud mental. Si bien la depresión postparto y la tristeza postnatal (o baby blues) pueden hacer parte del devenir del proceso puerperal, no es descartable que estas situaciones puedan verse incrementadas cuando hay VPI.

\section{CONFLICTO DE INTERESES}

Los autores declaran no tener ningún conflicto de intereses.
ISSN 1794-9831

E-ISSN 2322-7028

Vol. 12 No. 2

Jul - Dic 2015

Cúcuta, Colombia 
Violencia de la pareja íntima durante el embarazo y sus repercusiones en la salud mental - María Mercedes Lafaurie-Villamil, Lorena Liseth Cuadros-Salazar, Paola Andrea García-Bocanegra, Ingrid Johana Hernández-Ayala, Johanna Smit Pulido-Espinosa, Lina Fernanda Reynales-Triana, et al.

ISSN 1794-9831

E-ISSN 2322-7028 Vol. 12 No. 2 Jul - Dic 2015

\section{REFERENCIAS BIBLIOGRÁFICAS}

1. Organización Panamericana de la Salud. Prevención de la Violencia sexual y violencia infligida por la pareja contra las mujeres. Qué hacer y cómo obtener evidencias. Washington: OPS; 2011.

2. Organización de las Naciones Unidas. Declaración sobre la eliminación de la violencia contra la mujer. [Internet]. 1994 [consultado 12 de octubre de 2014] Disponible en: http:/www.ohchr.org/SP/ProfessionalInterest/Pages/ ViolenceAgainstWomen.aspx

3. Comisión Económica para América Latina y el Caribe (CEPAL). Violencia de género: un problema de derechos humanos. [Internet]. 1996 [consultado 20 de octubre de 2014]. Disponible en: http://www.cepal.org/publicaciones/ xml/5/4345/1c1957e.pdf

4. Páez-Cuba LD. La violencia de género: una sistematización Técnico-Jurídica. Biblioteca virtual de Derecho, Economía y Ciencias Sociales. [Internet]. 2011 [consultado 16 de septiembre de 2014]. Disponible en: http://www. eumed.net/librosgratis/2011a/925/Hacia\%20una\%20definicion\%20de\%20la\%20violencia\%20de\%20genero.htm

5. Organización de las Naciones Unidas. Conferencia Internacional sobre Población y Desarrollo. [Internet]. El Cairo: Organización de las Naciones Unidas; 1994 [consultado 13 de octubre de 2014]. Disponible en: http://www.un.org/ popin/icpd/newslett/94_19/icpd9419.sp/1lead.stx.html

6. Organización de las Naciones Unidas. IV Conferencia Mundial de la Mujer. [Internet]. Beijing: Naciones Unidas; 1995 [consultado 30 de octubre de 2014]. Disponible en: http://www.un.org/womenwatch/daw/beijing/pdf/ Beijing\%20full\%20report\%20S.pdf

7. Organización de las Naciones Unidas. Violencia contra las mujeres. La situación. [Internet]. Naciones Unidas; 2009 [consultado 30 de octubre de 2014]. Disponible en: http://www.un.org/es/events/endviolenceday/pdfs/unite_the_ situation_sp.pdf

8. Pérez-García C, Manzano-Felipe MA La práctica clínica enfermera en el abordaje de la violencia de género. Cultura de los cuidados [Internet]. 2014 [consultado 11 septiembre de 2014]; XVIII( 40):107-115. Disponible en: http://rua. ua.es/dspace/bitstream/10045/43947/1/Cultura-Cuidados_40_14.pdf

9. Lafaurie-Villamil MM. Violencia de la pareja íntima en relatos de gestantes atendidas en el Hospital de Usaquén (Bogotá, Colombia). Rev Colomb Enfer 2015; 10(11):45-56.

10. Ramírez EJ. Relación entre violencia de pareja contra la mujer y la aparición de depresión en el postparto. [Tesis magister]. Bogotá D.C.: Universidad Nacional de Colombia; 2012.

11. Núñez-Rivas HP, Monge-Rojas R, Gríos-Dávila C, Elizondo-Ureña AM, Rojas-Chavarría A. La violencia física, psicológica, emocional y sexual durante el embarazo: riesgo reproductivo predictor de bajo peso al nacer en Costa Rica. Rev Panam Salud Pública [Internet]. 2003 [consultado 10 mayo de 2015]; 14(2): 75-83. Disponible en: http:// www.scielosp.org/scielo.php?pid=S1020-49892003000700001\&script=sci_arttext

12. Lafaurie-Villamil MM. Intimate partner violence against women during pregnancy: a critical reading from a gender perspective. Rev Colomb Enferm. 2015; 10(10): 65-78.

13. Domínguez-Fuentes JM, García-Leiva P, Cuberos-Casado I. Violencia contra las mujeres en el ámbito doméstico: consecuencias sobre la salud psicosocial. Anales de psicología [Internet]. 2008 [consultado 26 de septiembre de 2014]; 24(1):115-120. Disponible en: http://www.um.es/analesps/v24/v24_1/14-24_1.pdf

14. Plazaola-Castaño J, Ruiz-Pérez I. Violencia contra la mujer en la pareja y consecuencias en la salud física y psíquica. MedClin (Barc) [Internet]. 2004 [consultado 26 de septiembre de 2014];122(12):461-7. Disponible en: http://www. catunescomujer.org/catunesco_mujer/documents/Art_Foro_Ruiz.pdf

15. Cuevas S, Blanco J, Juárez C, Palma O, Valdez-Santiago R. Violencia y embarazo en usuarias del sector salud en estados de alta marginación en México. Salud pública de México [Internet]. 2006 [consultado 20 de marzo de 2014]; 48(sup12):s239-s249 Disponible en: http://www.scielo.org.mx/pdf/spm/v48s2/31380.pdf.

16. Bonifaz R, Spano A. La violencia intrafamiliar, el uso de drogas en la pareja, desde la perspectiva de la mujer maltratada. Rev Latino-am. Enfermagem [Internet]. 2004 [consultado 20 de octubre de 2014]; 12 (número especial): 433-8. Disponible en: http://www.revistas.usp.br/rlae/article/viewFile/1984/2058

17. Castro R, Ruiz A. Prevalencia y severidad de la violencia contra mujeres embarazadas, México. Rev Saúde Pública 2004; 38(1):62-70.

18. Valladares E, Peña R, Persson LA, Högberg U. Violence against pregnant women: prevalence and characteristics. A population-based study in Nicaragua. BJOG 2005;112(9):1243-1248.

19. López S, Gómez-Sánchez PI, Arévalo-Rodríguez I. Violencia contra la mujer. Análisis en el Instituto Materno Infantil de Bogotá, Colombia, 2005. Estudio de corte transversal. Rev Colomb Obstet Ginecol 2007; 59(1): 10-19.

20. Doubova SV, Pámanes-González V, Billings D, Torres- Arreola LP. Violencia de pareja en mujeres embarazadas en la Ciudad de México. Rev Saúde Pública 2007; 41(4): 582-590. 
21. Medina-Meza P, Martínez-Salgado JC, Suarez-Rodríguez JC, Castro-Naranjo LJ, Muriel-Perea YY, Obando-Gómez NN. Violencia física y psicológica contra la mujer embarazada. Educ Invest Enf 2001;19(1): 18.

22. Taillieu $T$, Brownridge D. Violence against pregnant women: prevalence, patterns, risk factors, theories, and directions for future research. Aggression and Violent Behavior 2010; 15: 14-35.

23. Mendoza M, Martinez G, Pizano ML, Lartigue T. Violencia de género, embarazo y autoestima en un área urbana de la ciudad de México. Rev. Enferm. Herediana 2008; 01 (1):40-47.

24. Organización Mundial de la Salud OMS. Organización Panamericana de la Salud (OPS). Violencia infligida por la pareja. Comprender y abordar la violencia contra las mujeres. Oficina regional para las Américas [Internet]. s/f [consultado 20 de octubre de 2014]. Disponible en: http://www.paho.org/hq/index.php?option=com_ docman\&task $=$ doc_view\&gid $=23946 \&$ Itemid $=$

25. Howard LM, Oram S, Galley H, Trevillion K, Feder G. Domestic Violence and Perinatal Mental Disorders: A Systematic Review and Meta-Analysis. PLoS Med [Internet]. 2013 [consultado 20 de octubre de 2014]; 10(5):e1001452. Disponible en: http://www.plosmedicine.org/article/info\%3Adoi\%2F10.1371\%2Fjournal. pmed. 1001452

26. Farrokh H, Sima E, Negar H, Intimate partner violence during pregnancy in Urmia, Iran in 2012. Journal of Forensic and Legal Medicine [Internet]. 2014 [consultado 20 de octubre de 2014]; 24:28-32. Disponible en: http://dx.doi. org/10.1016/j.jflm.2014.03.007

27. Urquía M, O'Campo P, Heaman MI, Janssen PA, Thiessen K. Experiences of violence before and during pregnancy and adverse pregnancy outcomes: an analysis of the Canadian maternity experiences survey. BMC Pregnancy and Childbirth 2011; 11:42.

28. Izaguirre A, Calvete E, Intimate partner violence during pregnancy: Women's narratives about their mothering experiences, Psychosocial Intervention [Internet]. 2014 [consultado 20 de octubre de 2014];23(3):209-215. Disponible en: http://dx.doi.org/10.1016/j.psi.2014.07.010

29. Sánchez B, Hernández M, Lartigue T. Violencia conyugal y depresión durante el embarazo. Salud Pública Méx 2008; 50(5):353-354.

30. Castro R, Ruiz A. Prevalencia y severidad de la violencia contra mujeres embarazadas, México. Rev Saúde Pública 2004; 38(1):62-70.

31. Tavara-Orozco L, Zegarra-Samamé T, Huamaní S, Félix F, Espinoza-Tarazona K, Chumbe-Ruiz O, et al. Repercusiones maternas y perinatales de la violencia basada en género. Rev Per Ginecol Obstet 2007; 53:10-17.

32. McMahon S, Huang $\mathrm{C}$, Boxer $\mathrm{P}$, Postmus $\mathrm{L}$. The impact of emotional and physical: violence during pregnancy on maternal and child health at one year post-partum. Children and Youth Services. [Internet]. 2011 [consultado 8 de julio de 2014];33(11):2103-2111. Disponible en: https://www.researchgate.net/publication/251530618_The_ impact_of_emotional_and_physical_violence_during_pregnancy_on_maternal_and_child_health_at_one_year_post-partum

33. Peltzer K, Pengpid S, McFarlane J, Banyini M. Mental health consequences of intimate partner violence in Vhembe district, South Africa. General Hospital Psychiatry. [Internet]. 2013 [consultado 8 de julio de 2014];35(5):545-550. Disponible en: https://www.researchgate.net/publication/236637093_Mental_health_consequences_of_intimate_ partner_violence_in_Vhembe_district_South_Africa

34. Baird K, Saito A, Eustace J, Creedy D. An exploration of Australian midwives' knowledge of intimate partner violence against women during pregnancy. Women Birth [[Internet]. 2015 [consultado 8 de julio de 2014];28(3):21520. Disponible en: http://www.academia.edu/13373946/An_exploration_of_Australian_midwives_knowledge_ of_intimate_partner_violence_against_women_during_pregnancy

35. Ludermir $\mathrm{AB}$, Valongueiro $\mathrm{S}$, Barreto $\mathrm{T}$. Common mental disorders and intimate partner violence in pregnancy. Rev saúde Pública [Internet]. 2014 [consultado 04 de agosto de 2014];48(1):29-35. Disponible en: http://www. scielo.br/scielo.php?pid=S0034-89102014000100029\&script=sci_arttext

36. Ruíz-Pérez I. 3. Violencia contra la mujer y salud. [Internet]. España: Escuela Andaluza de Salud Pública; 2007 [consultado 08 de agosto de 2014]. Disponible en: http://www.msssi.gob.es/organizacion/sns/planCalidadSNS/pdf/ equidad/04modulo_03.pdf

37. Escobar MR. Estado del arte del conocimiento producido sobre jóvenes en Colombia 1985-2003. Bogotá: Programa Presidencial Colombia Joven - Agencia de Cooperación Alemana GTZ - UNICEF Colombia; 2004.

38. Universidad de Antioquia. Seminario Estudios de Usuarios. Medellin: Universidad de Antioquia; 2003.

39. Montero M. Hochman Investigación Documental, Técnicas y procedimientos. Caracas: Editorial Panapo; 2005.

40. Republica de Colombia. Ministerio de Salud. Resolución 008430 de 1993, octubre 4, por la cual se establecen las normas científicas, técnicas y administrativas para la investigación en salud. [Internet]. Santa de Bogota: Ministerio de Salud; 1993 [consultado 8 de noviembre de 2013]. Disponible en: http://www.urosario.edu.co/urosario_files/ 
Violencia de la pareja íntima durante el embarazo y sus repercusiones en la salud mental • María Mercedes Lafaurie-Villamil, Lorena Liseth Cuadros-Salazar, Paola Andrea García-Bocanegra, Ingrid Johana Hernández-Ayala, Johanna Smit Pulido-Espinosa, Lina Fernanda Reynales-Triana, et al.

ISSN 1794-9831

E-ISSN 2322-7028 Vol. 12 No. 2 Jul - Dic 2015 a2/a24fb07a-f561-4fcc-b611-affff4374bb7.pdf

41. Congreso de Colombia. Ley 911 de 2004, octubre 6, por la cual se dictan disposiciones en materia de responsabilidad deontológica para el ejercicio de la profesión de Enfermería en Colombia; se establece el régimen disciplinario correspondiente y se dictan otras disposiciones. [Internet].Santa Fe de Bogota: Ministerio de la Proteccion Social; 1993 [consultado 6 de noviembre de 2013]. Disponible en: http://www.trienfer.org.co/index.php?option=com_con tent\&view $=$ article\&id=19\&Itemid $=27$

42. Tiwari A, Chan K, Fong D, Leung W, Brownridge D, Lam H, et al. The impact of psychological abuse by an intimate partner on the mental health of pregnant women. BJOG [Internet]. 2008 [consultado 07 de noviembre de 2013];115(3): 377-384. Disponible en: http://www.ncbi.nlm.nih.gov/pmc/articles/PMC2253706/

43. Zhang Y, Zou S, Cao Y, Zhang Y. Relationship between domestic violence and postnatal depression among pregnant Chinese women, International Journal of Gynecology and Obstetrics [Internet]. 2012 [consultado 07 de noviembre de 2013];116(1):26-30. Disponible en: http://dx.doi.org/10.1016/j.ijgo.2011.08.011

44. Varma B, Prabha S, Tinku T, Carey M. Intimate partner violence and sexual coercion among pregnant women in India: Relationship with depression and post-traumatic stress disorder. Journal of Affective Disorders [Internet]. 2007 [consultado 07 de noviembre de 2013];102(1-3):227-235. Disponible en: http://dx.doi.org/10.1016/j. jad.2006.09.026

45. Groves AK, Kagee A, Maman S, Moodley D, Rouse P. Associations between intimate partner violence and emotional distress among pregnant women in Durban, South Africa. J Interpers Violence 2012;27(7):1341-1356.

46. Mahenge B, Likindikoki S, Stöckl H, Mbwambo J. Intimate partner violence during pregnancy and associated mental health symptoms among pregnant women in Tanzania: a cross-sectional study. BGOG 2013;120(8):940946.

47. Lam N, Contreras H, Cuesta F, Mori E, Cordori J, Carrillo N. Resiliencia y apoyo social frente a trastornos depresivos en gestantes sometidas a violencia de género. Revista Peruana de Epidemiología [Internet]. 2008 [consultado 07 de noviembre de 2013];12(3)1-8. Disponible en: http://www.redalyc.org/articulo.oa?id=203120335005

48. Gomez A, Williams M, Sanchez S, Lam N. Intimate partner violence and risk for depression among postpartum women in Lima, Peru. Violence Vict 2009; 24(3):380-98.

49. Cowley D. Later Effects of Psychological Abuse During Pregnancy. Journal Watch [Internet]. 2010 [consultado 07 de noviembre de 2013];30(19). Disponible en: http://www.medscape.com/viewarticle/731350

50. Nunes M, Ferri C, Manzolli $P$, Soares R, Drehmer M, Buss C, et al. Nutrition, mental health and violence: from pregnancy to postpartum Cohort of women attending primary care units in Southern Brazil - ECCAGE study. BMC Psychiatry [Internet]. 2010 [consultado 8 de febrero de 2015];10(66). Disponible en: http://goo.gl/MA8x6x

51. Santos-Mota R, Matías-Santos M, Camargo C, Pereira-Gomes N, Freire-Diniz N, Diniz-Rodrigues A. Sexualidad, embarazo y violencia doméstica: experiencias de adolescentes brasileñas. Index Enferm [Internet]. 2014 [consultado 18 de febrero de 2015];23(3):139-143. Disponible en: http://scielo.isciii.es/scielo.php?pid=S1132$12962014000200005 \&$ script $=$ sci_arttext

52. Zabala-González M, Bautista-Torres J, Quebedo-Tejeiro E. Depresión en mujeres con perimenopausia de una comunidad rural de Huimanguillo, Tabasco, México. Archivos de Medicina 2009, 5 (4): 171-177.

53. Jadresic E. Depresión en el embarazo y el puerperio. Rev. chil. neuro-psiquiatr. [Internet]. 2010 [consultado 18 de diciembre de 2015]; 48(4):269-278. Disponible en: http:/www.scielo.cl/scielo.php?script=sci_arttext\&pid=S071792272010000500003\&lng=es. http://dx.doi.org/10.4067/S0717-92272010000500003.

54. Sánchez-Montoya J, Palacios-Alzaga G. Trastorno de estrés postraumático en el embarazo, parto y posparto. Matronas Prof. 2007; 8(1):12-19.

55. Asociación Americana de Psquiatría. Criterios diagnósticos del DSM 5. Washington: American Psychiatric Publishing; 2014.

56. Maldonado-Durán $M$, Lartigue $T$. Trastornos de ansiedad en la etapa perinatal. Perinatol Reprod Hum [Internet]. 2008 [consultado 19 de diciembre de 2015]; 22:100-110. Disponible en: http://www.inper.edu.mx/descargas/pdf/ Trastornosdeansiedad.pdf

57. Cataño-Toro D, Restrepo S A, Portilla ND, Ramírez HD. Autoestima y sexualidad en adolescentes: validación de una escala. Investigaciones Andina 2008;10(16): 57-66.

58. Matud MP. Autoestima en la mujer,. Un análisis de su relevancia en la salud. Avances en Psicología Latinoamericana 2004; $22: 129-140$.

59. Organización Mundial de la Salud (OMS). Violencia contra la mujer [Internet]. 2014 [consultado 19 de diciembre de 2014]. Disponible en: http://www.who.int/mediacentre/factsheets/fs239/es/ 